\title{
Physiological parameter values in greyhounds before and after high-intensity exercise
}

\author{
Francisco Javier Pellegrino ${ }^{1,2,3}$, Analía Risso ${ }^{1,2,3}$, Pablo G. Vaquero ${ }^{4}$ and Yanina A. Corrada ${ }^{1,2, *}$ \\ ${ }^{1}$ LAFIVET - Laboratorio de Fisioterapia Veterinaria, Facultad de Ciencias Veterinarias, Universidad Nacional de \\ La Plata, 60 y 118 (1900) La Plata, Argentina \\ ${ }^{2}$ Consejo Nacional de Investigaciones Científicas y Técnicas, (CONICET LA PLATA), $8 N^{\circ}$ 1467, La Plata, \\ Argentina \\ ${ }^{3}$ IGEVET - Instituto de Genética Veterinaria (UNLP-CONICET LA PLATA), 60 y 118 (1900) La Plata, Argentina \\ ${ }^{4}$ Facultad de Ciencias Veterinarias, Universidad Nacional de La Pampa (UNLPam), 5 esq. 116 (6360), General \\ Pico, La Pampa, Argentina
}

\begin{abstract}
Dog sports competitions have greatly expanded. The availability of reference values for each type of activity could help assess fitness accurately. Heart rate (HR), blood lactate (BL) and rectal temperature (RT) are relevant physiological parameters to determine the dogs response to effort. Previous studies in greyhounds have reported the effect of high-intensity exercise on many physiological parameters immediately after completing different racing distances and recovery times. However, there are no studies concerning physiological changes over shorter racing distances. We therefore assessed the effect of sprint exercise on HR, BL and RT in nine greyhounds performing sprint exercise over a 100-m distance chasing a lure. After the exercise, dogs underwent a passive 10-min recovery phase. Before the exercise, immediately after it and at 5 and 10 min during recovery, HR and RT were assessed and blood samples were collected for BL determination. HR, BL and RT values increased significantly after the exercise $(\mathrm{P}<0.01)$. Whereas HR returned to pre-exercise values at 10 min during the recovery phase $(\mathrm{P}>0.1), \mathrm{BL}$ concentration and RT remained increased $(\mathrm{P}<0.01)$. The abrupt increase in HR, BL and RT values observed immediately after the exercise indicates the high intensity of the effort performed. Similarly, BL concentration after the exercise exceeded the $4 \mathrm{mmol} / \mathrm{L}$ lactate threshold, suggesting a predominant anaerobic metabolism during effort. Although HR returned to pre-exercise values $10 \mathrm{~min}$ after the exercise, a more extensive recovery phase would be necessary for a total return to resting values, particularly for BL and RT. In greyhounds subjected to high-intensity exercise, HR, BL and RT were reliable physiological parameters to accurately assess the physiological response to effort. The use of sprint exercises over short racing distances could be useful for appropriately monitoring fitness in sporting dogs.
\end{abstract}

Keywords: Blood lactate, Exercise, Greyhound, Heart rate, Rectal temperature.

\section{Introduction}

Over the last 30 years, dog sports competitions have greatly expanded (Wakshlag and Shmalberg, 2014). Their growing popularity has led to an increase in the number of scientific reports aimed at explaining the physiological changes caused by exercise (Angle et al., 2009), as well as the time required to achieve resting values after exercise (Rovira et al., 2007; Baltzer et al., 2012).

In canine athletes, reference values for each type of activity would help assess fitness accurately, as the response varies according to the type, duration and intensity of the effort performed (Piccione et al., 2012). Among the physiological parameters assessed, heart rate $(\mathrm{HR})$, blood lactate $(\mathrm{BL})$ and rectal temperature (RT) are relevant measurements to evaluate the effort required by the exercise (Ferasin and Marcora, 2009). In sports medicine, the term "lactate threshold" is typically used to show the transition point between aerobic and anaerobic metabolism. In humans, it has been established at $4 \mathrm{mmol} / \mathrm{L}$ BL concentration (Ghosh, 2004). Exercise intensity exceeding the threshold will demand a greater intake of carbohydrates and a larger contribution of anaerobic metabolism to obtain energy, with muscle lactate production being proportional to the intensity of the effort (Rovira et al., 2007).

In contrast, exercise intensity below the threshold demands a larger contribution of aerobic metabolism, mainly depending on carbohydrates and fats for the production of energy (Rovira et al., 2007). Thus, during high-intensity exercises the maximum consumption of oxygen provided by aerobic metabolism is exceeded, so that a predominant anaerobic metabolism is required as a source of energy (Crisafulli et al., 2004). Therefore, BL concentration during exercise would indicate the 
type of effort performed. Previous studies in greyhounds have reported the effect of high-intensity exercise on many physiological parameters immediately after completing different racing distances and recovery times (Snow et al., 1988; Ilkiw et al., 1989; Rose and Bloomberg, 1989; Holloway et al., 1996). These studies have shown the marked increase in $\mathrm{HR}, \mathrm{BL}$ and $\mathrm{RT}$ values as well as in other biochemical and hematological parameters after finishing the exercise.

However, there are currently no studies available concerning the physiological changes associated with exercise over shorter racing distances. On the other hand, the return of the parameters assessed during the recovery phase to pre-exercise values may be useful to evaluate the training level in sporting dogs.

It has been proposed that decreases in BL concentration during the recovery phase could suggest appropriate adaptation of the dogs to the effort performed (Rovira et al., 2007). Therefore, the aim of this study was to assess the effect of sprint exercise on HR, BL and RT in greyhounds.

\section{Materials and Methods}

\section{Animals}

The study was approved by the Institutional Animal Care and Use Committee (No. T30-1-12) of the School of Veterinary Sciences, National University of La Plata, Buenos Aires, Argentina. Nine healthy greyhounds were included in this study (male, $\mathrm{n}=3$; female, $\mathrm{n}=6$; age range, 2-5 y; weight, $25-33 \mathrm{~kg}$; height at withers, $59-70 \mathrm{~cm}$ ). Before the study, all dogs were subjected to a full clinical and orthopedic examination.

\section{Exercise protocol}

All the dogs performed a sprint exercise over a 100-m distance, chasing a lure. After completing the exercise, the dogs underwent a passive 10-min recovery phase during which they remained at rest or in sternal recumbency.

\section{Measurements}

All measurements were taken before (Pre) and immediately after (Post) the exercise, and at 5 (Post 5$\mathrm{min}$ ) and $10 \mathrm{~min}$ (Post 10-min) during the recovery phase. HR was determined by cardiac auscultation and expressed as beats per min (bpm). Venous blood samples (approximately $50 \mu \mathrm{l}$ ) were obtained by venipuncture of the saphenous vein to assess $\mathrm{BL}$ concentration $(\mathrm{mmol} / \mathrm{L})$. Individual lactate measurements were performed immediately after collecting the samples, using Nova Biomedical StatStrip X-Press ${ }^{\circledR}$ lactate enzymatic-colorimetric analyzer (Bonaventura et al., 2015). A rapid reading digital thermometer was used to measure RT in ${ }^{\circ} \mathrm{C}$.

\section{Statistical analysis}

We used an experimental design with repeated measures in time. Each individual dog was considered an experimental unit. Data were analyzed using the
Proc Mixed of SAS (version 9.0; SAS Institute Inc., Cary, NC, USA). The linear mixed model included the random effect of dogs and the fixed effect of time (Pre vs. Post vs. Post 5-min vs. Post 10-min). Data are represented as least square means (LSM) \pm the standard error of the means (SEM). For the main effect (time) the alpha level of significance was set at $\mathrm{P}<0.05$.

\section{Results and Discussion}

In our study, HR, BL and RT values increased significantly after the exercise $(\mathrm{P}<0.01)$. While HR remained increased at $5 \mathrm{~min}$ of the recovery phase $(\mathrm{P}<0.01)$, it returned to pre-exercise values at $10 \mathrm{~min}$ $(\mathrm{P}>0.1)$. BL concentration and $\mathrm{RT}$ remained increased both at 5 and at 10 min during the recovery phase $(\mathrm{P}<0.01)$. The highest $\mathrm{BL}$ and $\mathrm{RT}$ values were reported 5 min after finishing the exercise (Table 1; Figures 1$3)$.

Table 1. Physiological parameters evaluated before (Pre), immediately after (Post) and at 5 (Post 5-min) and $10 \mathrm{~min}$ (Post 10-min) following a sprint exercise in 9 greyhounds.

\begin{tabular}{|c|c|c|c|c|c|}
\hline \multirow{2}{*}{ Parameter } & Pre & Post & $\begin{array}{c}\text { Post } \\
\text { 5-min }\end{array}$ & $\begin{array}{c}\text { Post } \\
10-\text { min } \\
\end{array}$ & \multirow{2}{*}{ SEM } \\
\hline & LSM & LSM & LSM & LSM & \\
\hline $\begin{array}{c}\text { Heart rate } \\
(\mathrm{bpm})\end{array}$ & $139.10^{\mathrm{a}}$ & $169.33^{\mathrm{b}}$ & $154.22^{\mathrm{c}}$ & $144.00^{\mathrm{a}}$ & 3.46 \\
\hline $\begin{array}{l}\text { Blood lactate } \\
(\mathrm{mmol} / \mathrm{L})\end{array}$ & $1.70^{\mathrm{a}}$ & $8.58^{\mathrm{b}}$ & $9.08^{\mathrm{b}}$ & $6.54^{c}$ & 0.95 \\
\hline $\begin{array}{l}\text { Rectal temp. } \\
\quad\left({ }^{\circ} \mathrm{C}\right)\end{array}$ & $39.32^{\mathrm{a}}$ & $40.31^{\mathrm{b}}$ & $40.45^{b}$ & $40.31^{\mathrm{b}}$ & 0.22 \\
\hline
\end{tabular}

Values are expressed as LSM \pm SEM. Different superscripts indicate differences between times $(\mathrm{P}<0.05)$.

The assessed HR and RT values before starting the exercise probably reflect an anticipatory response among the dogs under study (Steiss et al., 2004), given the potential anxiety to start the exercise. However, this does not seem to have influenced the values obtained after the exercise, considering the significant increase observed in the parameters assessed. The abrupt increase in $\mathrm{HR}$, BL and RT values immediately after the exercise indicates the high-intensity of the effort performed. At 5 min during the recovery phase, HR values significantly decreased as compared with those obtained immediately after the exercise. However, they remained high as compared with those assessed before starting the exercise. In contrast, at $10 \mathrm{~min}$ during the recovery phase, HR returned to pre-exercise values, suggesting an appropriate recovery of the dogs after the exercise. The increased BL concentration at the end of the high-intensity exercise is mainly due to lactate release from the muscles previously involved in the effort (Maciejewski et al., 2016). In this study, BL concentration in all dogs after exercise exceeded the lactate threshold of $4 \mathrm{mmol} / \mathrm{L}$, suggesting a predominant anaerobic metabolism in muscle contraction during effort. 


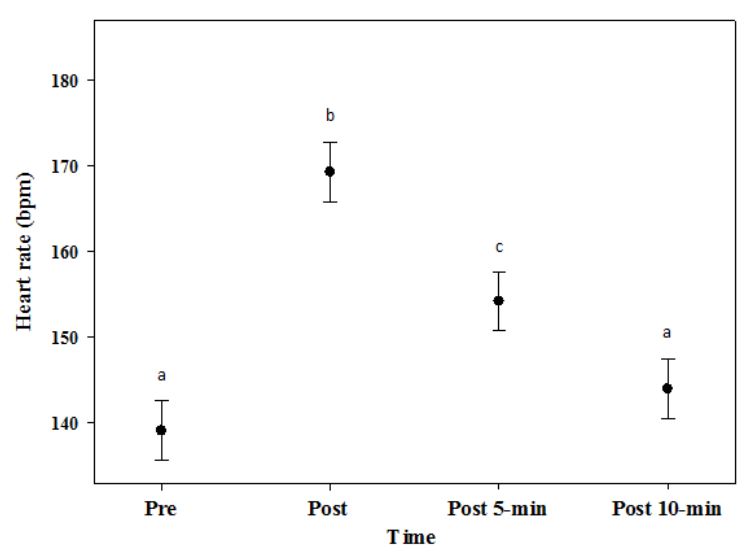

Fig. 1. Heart rate (bpm) before (Pre), immediately after (Post) and at 5 (Post 5-min) and 10 min (Post 10-min) following a high-intensity exercise in 9 greyhounds. Values are expressed as LSM \pm SEM. Different superscripts indicate differences between times $(\mathrm{P}<0.05)$.

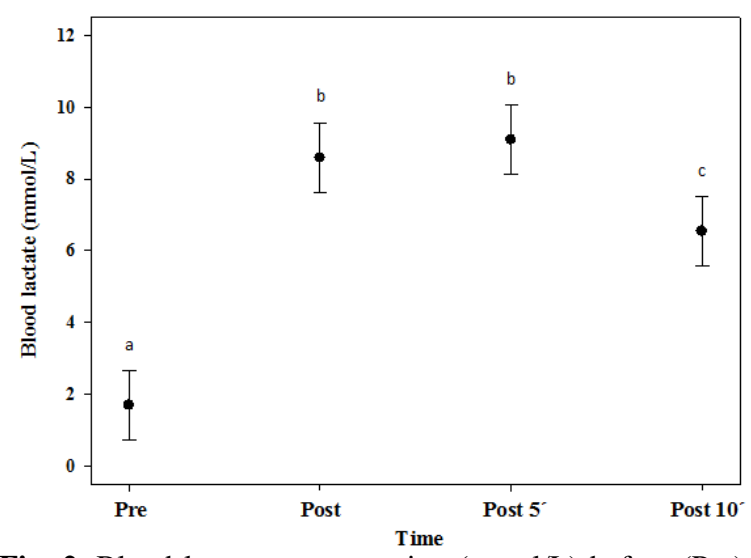

Fig. 2. Blood lactate concentration ( $\mathrm{mmol} / \mathrm{L})$ before (Pre), immediately after (Post) and at 5 (Post 5-min) and $10 \mathrm{~min}$ (Post 10-min) following a high-intensity exercise in 9 greyhounds. Values are expressed as LSM \pm SEM. Different superscripts indicate differences between times $(\mathrm{P}<0.05)$.

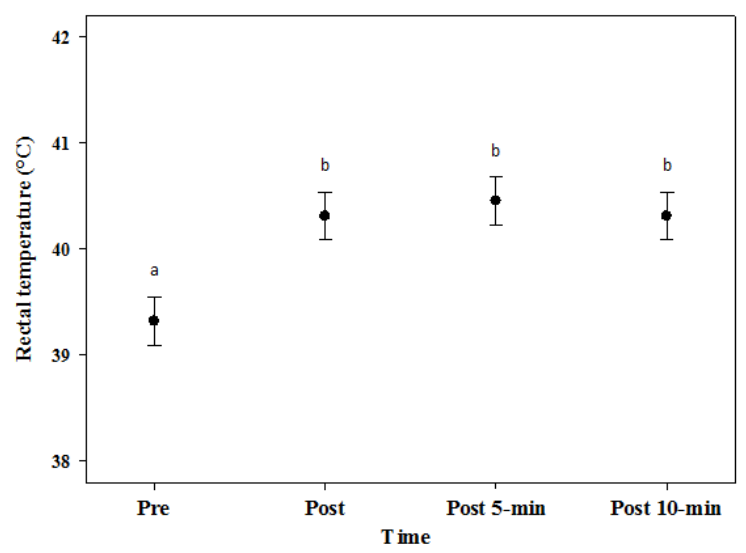

Fig. 3. Rectal temperature $\left({ }^{\circ} \mathrm{C}\right)$ before (Pre), immediately after (Post) and at 5 (Post 5-min) and $10 \mathrm{~min}$ (Post 10-min) following a high-intensity exercise in 9 greyhounds. Values are expressed as LSM \pm SEM. Different superscripts indicate differences between times $(\mathrm{P}<0.05)$.
A previous study in greyhounds showed that this breed had a larger percentage of fast-twitch type-IIA muscle fibers in their limbs (Guy and Snow, 1981). In a more recent study, the types of muscle fibers making up the muscles of various dog breeds were determined, showing that type-IIA fiber was the most abundant in the muscles of the trunk and limbs (Toniolo et al., 2007). Since these fibers have a remarkable glycolytic capacity, they are capable of producing large quantities of lactate during high-intensity exercises (Essén et al., 1975). Although in the present study we did not assess the relative quantity of the types of muscle fibers in our sample of dogs, they would have probably shown a larger proportion of type-II muscle fibers, which may have led to increased $\mathrm{BL}$ at the end of the exercise. The increased BL concentration during the first minutes of recovery is due to the fact that the presence of lactate in blood temporarily exceeds its elimination (Maciejewski et al., 2016). Assuming that lactate production is interrupted once the effort ends, the peak reported in BL values at $5 \mathrm{~min}$ during the recovery phase can be the result of a delayed release of lactate from the muscles to the blood flow. This could be caused by a low density of the proteins involved in the transportation of lactate in muscles, and/or by a limited intracellular metabolism (Ferasin and Marcora, 2009). Our results are in line with those previously reported in greyhounds, in which the peak in BL concentration after high-intensity exercise occurred at 5 min during recovery, while the return to pre-exercise values did not occur until after $30 \mathrm{~min}$ following the exercise (Rose and Bloomberg, 1989). In turn, RT showed a similar behavior to BL during the recovery phase. Muscle activity generates heat as a byproduct of the production and use of adenosine triphosphate. Previous studies in dogs have reported increases in RT values after exercise (Kozłowski et al., 1985; Ilkiw et al., 1989; Matwichuk et al., 1999; Steiss et al., 2004).

In greyhounds, muscle mass accounts for approximately $60 \%$ of the total body weight, so it is expected that a high-intensity exercise may generate heat at a fast pace (McNicholl et al., 2016). Similarly, ambient temperature may have influenced body temperature after exercise (McNicholl et al., 2016), conditioning the process of thermoregulation during recovery. Although a mild decrease was observed in mean BL and RT values at 10 min during the recovery phase, these proved significantly higher than those obtained before starting the exercise. In this sense, other studies in dogs reported longer recovery times so that the physiological parameters assessed may return to pre-exercise values after high-intensity exercises (Ilkiw et al., 1989; Rose and Bloomberg, 1989).

\section{Conclusion}

In greyhounds subjected to high-intensity exercise, HR, $\mathrm{BL}$ and $\mathrm{RT}$ are reliable physiological parameters to 
accurately assess the physiological response of dogs to effort. Based on the results obtained in this study, a more extensive recovery phase would be necessary for a total return of the assessed parameters to resting values, particularly BL concentration and RT. Finally, the use of sprint exercises over short racing distances could be useful for appropriately monitoring fitness in sporting dogs, mainly during the precompetitive phase.

\section{Acknowledgments}

The study was partially funded by the project V-241 from Proyecto de Incentivos, Universidad de La Plata, Buenos Aires, Argentina. We would like to thank A. Di Maggio for manuscript editing and technical assistance. Conflict of interest

The authors declare that there is no conflict of interests.

\section{References}

Angle, C.T., Wakshlag, J.J., Gillette, R.L., Stokol, T., Geske, S., Adkins, T.O. and Gregor, C. 2009. Hematologic, serum biochemical, and cortisol changes associated with anticipation of exercise and short duration high-intensity exercise in sled dogs. Vet. Clin. Pathol. 38(3), 370-374.

Baltzer, W.I., Firshman, A.M., Stang, B., Warnock, J.J., Gorman, E. and McKenzie, E.C. 2012. The effect of agility exercise on eicosanoid excretion, oxidant status, and plasma lactate in dogs. BMC Vet. Res. 8, 249.

Bonaventura, J.M., Sharpe, K., Knight, E., Fuller, K.L., Tanner, R.K. and Gore, C.J. 2015 Reliability and accuracy of six hand-held blood lactate analysers. J. Sports Sci. Med. 14(1), 203-214.

Crisafulli, A., Carta, C., Melis, F., Tocco, F., Frongia, F., Santaboni, U.M., Pagliaro, P. and Concu, A. 2004. Haemodynamic responses following intermittent supramaximal exercise in athletes. Exp. Physiol. 89(6), 665-674.

Essén, B., Jansson, E., Henriksson, J., Taylor, A.W. and Saltin, B. 1975. Metabolic characteristics of fibre types in human skeletal muscle. Acta Physiol. Scand. 95(2), 153-165.

Ferasin, L. and Marcora, S. 2009. Reliability of an incremental exercise test to evaluate acute blood lactate, heart rate and body temperature responses in Labrador retrievers. J. Comp. Physiol. B. 179(7), 839-845.

Ghosh, A.K. 2004. Anaerobic threshold: its concept and role in Endurance sport. Malays J. Med. Sci. 11(1), 24-36.

Guy, P.S. and Snow, D.H. 1981. Skeletal muscle fibre composition in the $\mathrm{dog}$ and its relationship to athletic ability. Res. Vet. Sci. 31(2), 244-248.

Holloway, S.A., Sundstrom, D. and Senior, D.F. 1996. Effect of acute induced metabolic alkalosis on the $\mathrm{acid} / \mathrm{b}$ ase responses to sprint exercise of six racing greyhounds. Res. Vet. Sci. 61(3), 245-251.

Ilkiw, J.E., Davis, P.E. and Church, D.B. 1989. Hematologic, biochemical, blood-gas, and acidbase values in greyhounds before and after exercise. Am. J. Vet. Res. 50(4), 583-586.

Kozłowski, S., Brzezińska, Z., Kruk, B., KaciubaUściłko, H., Greenleaf, J.E. and Nazar, K. 1985. Exercise hyperthermia as a factor limiting physical performance: temperature effect on muscle metabolism. J. Appl. Physiol. 59(3), 766-773.

Maciejewski, H., Bourdin, M., Féasson, L., Dubouchaud, A., Denis, C., Freund, H. and Messonnier, L.A. 2016. Muscle MCT4 content is correlated with the lactate removal ability during recovery following all-out supramaximal exercise in highly-trained rowers. Front. Physiol. 7, 223.

Matwichuk, C.L., Taylor, S., Shmon, C.L., Kass, P.H. and Shelton, G.D. 1999. Changes in rectal temperature and hematologic, biochemical, blood gas, and acid-base values in healthy Labrador Retrievers before and after strenuous exercise. Am. J. Vet. Res. 60(1), 88-92.

McNicholl, J., Howarth, G.S. and Hazel, S.J. 2016. Influence of the environment on the body temperature of racing Greyhounds. Front. Vet. Sci. 3,53 .

Piccione, G., Casella, S., Panzera, M., Giannetto, C. and Fazio, F. 2012. Effect of moderate treadmill exercise on some physiological parameters in untrained beagle dogs. Exp. Anim. 61(5), 511-515.

Rose, R.J. and Bloomberg, M.S. 1989. Responses to sprint exercise in the greyhound: effects on haematology, serum biochemistry and muscle metabolites. Res. Vet. Sci. 47(2), 212-218.

Rovira, S., Muñoz, A. and Benito, M. 2007. Hematologic and biochemical changes during canine agility competitions. Vet. Clin. Pathol. 36(1), 30-35.

Snow, D.H., Harris, R.C. and Stuttard, E. 1988. Changes in haematology and plasma biochemistry during maximal exercise in greyhounds. Vet. Rec. 123(19), 487-489.

Steiss, J., Ahmad, H.A., Cooper, P. and Ledford, C. 2004. Physiologic responses in healthy Labrador retrievers during field trial training and competition. J. Vet. Intern. Med. 18(2), 147-151.

Toniolo, L., Maccatrozzo, L., Patruno, M., Pavan, E., Caliaro, F., Rossi, R., Rinaldi, C., Canepari, M., Reggiani, C. and Mascarello, F. 2007. Fiber types in canine muscles: myosin isoform expression and functional characterization. Am. J. Physiol. Cell Physiol. 292(5), C1915-C1926.

Wakshlag, J. and Shmalberg, J. 2014. Nutrition for working and service dogs. Vet. Clin. North Am. Small Anim. Pract. 44(4), 719-740. 